Notre Dame Journal of Formal Logic

Volume XVI, Number 1, January 1975

NDJFAM

\title{
A NEW POSTULATE-SYSTEM FOR MODULAR LATTICES
}

\section{BOLESŁAW SOBOCIŃSKI}

In [1], p. 384, Theorem 2, Milan Kolibiar has proved that the following two formulas

$A 1[a b c d]: a, b, c, d \in A . \supset .((a \cap b) \cap c) \cup(a \cap d)=((d \cap a) \cup(c \cap b)) \cap a$

and

A2 $[a b]: a, b \in A . \supset .(a \cup(b \cap b)) \cap b=b$

constitute an adequate postulate-system for modular lattices. It remains an open problem, $c f .,[1]$, p. 386, Remark, whether Kolibiar's postulatesystem can be sustituted by a shorter one.*

In this note a positive answer will be given for this problem. Namely, it will be proved:

(A) Any algebraic system

$$
\mathfrak{A}=\langle A, \cup, \cap\rangle
$$

where $\cup$ and $\cap$ are two binary operations defined on the carrier set $A$, is a modular lattice, if it satisfies the following two mutually independent postulates:

$B 1 \quad[a b c]: a, b, c \in A . \supset .(a \cap b) \cup(a \cap c)=((c \cap a) \cup(b \cup b)) \cap a$

B2 $[a b c]: a, b, c \in A . \supset . a=(c \cup(b \cup a)) \cap a$

Proof:

1 It is self-evident that $B 1$ and $B 2$ are provable formulas in the field of any modular lattice. In order to prove the converse let us assume $B 1$ and $B 2$. Then:

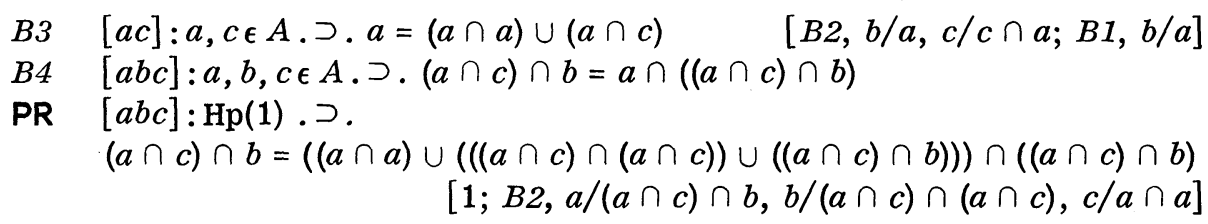

\footnotetext{
*See final remark, NB, at the end of this paper.
} 


$$
\begin{aligned}
& =((a \cap a) \cup(a \cap c)) \cap((a \cap c) \cap b) \\
& =a \cap((a \cap c) \cap b)
\end{aligned}
$$

$[B 3, a / a \cap c, c / b]$

[B3]

B5 $[a b c]: a, b, c \epsilon A . \supset .(a \cap(b \cap b)) \cup(a \cap c)=((c \cap a) \cup b) \cap a$

PR $\quad[a b c]: \operatorname{Hp}(1) . \supset$.

$(a \cap(b \cap b)) \cup(a \cap c)=((c \cap a) \cup((b \cap b) \cup(b \cap b))) \cap a$

$$
=((c \cap a) \cup b) \cap a
$$

$[1 ; B 1, b / b \cap b]$

$[B 3, a / b, c / b]$

B6 $[a b]: a, b \in A . \supset . a \cap a=((a \cap b)) \cap(a \cap b)) \cup(a \cap a)$

PR $[a b]: \operatorname{Hp}(1) . \supset$.

$a \cap a=((a \cap a) \cup(a \cap b)) \cap a=(a \cap((a \cap b) \cap(a \cap b))) \cup(a \cap a)$

$$
\cdot=((a \cap b) \cap(a \cap b)) \cup(a \cap a)
$$

$[1 ; B 3, c / b ; B 5, b / a \cap b, c / a]$

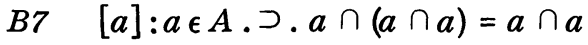

$[B 4, c / b, b / a \cap b]$

PR $[a]: \operatorname{Hp}(1) . \supset$.

$$
\begin{array}{rrr}
a \cap(a \cap a) & =((a \cap a) \cup(a \cap a)) \cap(a \cap a) & {[1 ; B 3, c / a]} \\
& =((a \cap a) \cup(((a \cap a) \cap(a \cap a)) \cup(a \cap a))) \cap(a \cap a) & {[B 6, b / a]} \\
& =a \cap a & {[B 2, a / a \cap a, b /(a \cap a) \cap(a \cap a), c / a \cap a]}
\end{array}
$$

B8 $[a]: a \in A . \supset .(a \cap a) \cap(a \cap a)=((a \cap a) \cap(a \cap a)) \cup((a \cap a) \cap(a \cap a))$

PR $[a]: \operatorname{Hp}(1) . \supset$.

$$
\begin{aligned}
(a \cap a) \cap(a \cap a) & =(((a \cap a) \cap(a \cap a)) \cup(a \cap a)) \cap(a \cap a) \quad[1 ; B 6, b / a] \\
& =((a \cap a) \cap((a \cap a) \cap(a \cap a))) \cup((a \cap a) \cap(a \cap a)) \\
& \quad[((a \cap a) \cap(a \cap a)) \cup((a \cap a) \cap(a \cap a)) \quad[B 7, a / a \cap a]
\end{aligned}
$$

B9 $[a]: a \in A . \supset .(a \cap a) \cap(a \cap a)=a \cap a$

PR $[a]: \operatorname{Hp}(1) . \supset$.

$$
\begin{array}{rrr}
(a \cap a) \cap(a \cap a) & =((a \cap a) \cap(a \cap a)) \cup((a \cap a) \cap(a \cap a)) & {[1 ; B 8]} \\
& =(((a \cap a) \cap(a \cap a)) \cup((a \cap a) \cup(a \cap a))) \cap(a \cap a) \\
& =a \cap a & {[B 1, a / a \cap a, b / a \cap a, c / a \cap a]} \\
& \quad[B 2, a / a \cap a, b / a \cap a, c /(a \cap a) \cap(a \cap a)]
\end{array}
$$

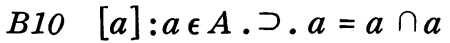

PR $[a]: \operatorname{Hp}(1) . \supset$.

$a=(a \cap a) \cup(a \cap a)=((a \cap a) \cap(a \cap a)) \cup((a \cap a) \cap(a \cap a))$

$$
=(a \cap a) \cap(a \cap a)=a \cap a
$$

$[1 ; B 3, c / a ; B 9 ; B 9]$

$[B 8 ; B 9]$

$B 11[a]: a \in A . \supset . a=a \cup a$

$[B 3, c / a ; B 10 ; B 10]$

$B 12[a b c]: a, b, c \epsilon A . \supset .(a \cap b) \cup(a \cap c)=((c \cap a) \cup b) \cap a \quad[B 1 ; B 11, a / b]$

$B 13[a b]: a, b \in A . \supset . a=(b \cup a) \cap a$

$B 14[a b]: a, b \in A$. ว. $a \cap b=a \cap(a \cap b)$

PR $[a b]: \operatorname{Hp}(1) . \supset$.

$$
\begin{aligned}
a \cap b & =((a \cap a) \cup(a \cap b)) \cap(a \cap b) \\
& =(((b \cap a) \cup a) \cap a) \cap(a \cap b) \\
& =a \cap(a \cap b)
\end{aligned}
$$

$[B 2, b / a, c / b ; B 11]$

$[a b]: a, b \in A, \supset, a=a \cup(a \cap b)$

$B 16[a b]: a, b \in A . \supset . a=(a \cap b) \cup a$

PR $[a b]: \mathrm{Hp}(1) . \supset$.

$a=a \cap a=((a \cap a) \cup(a \cap b)) \cap a$

$$
=(a \cap(a \cap b)) \cup(a \cap a)=(a \cap b) \cup a
$$

$[1 ; B 13, a / a \cap b, b / a \cap a]$ $[B 12, b / a, c / b]$ $[B 13, b / b \cap a]$ $[B 3, c / b ; B 10]$

$[1 ; B 10 ; B 3, c / b]$ $[B 12, b / a \cap b, c / a ; B 14 ; B 10]$ 
$B 17[a b]: a, b \in A . \supset . a \cap b=b \cap a$

PR $[a b]: \mathrm{Hp}(1) . \supset$.

$a \cap b=(a \cap b) \cup(a \cap b)=((b \cap a) \cup b) \cap a \quad[1 ; B 11, a / a \cap b ; B 12, c / b]$

$$
=b \cap a
$$

$[B 16, a / b, b / a]$

$B 18[a b]: a, b \in A . \supset . a=(a \cup b) \cap a$

PR $[a b]: \mathrm{Hp}(1) . \supset$.

$a=(a \cap b) \cup a=(a \cap b) \cup(a \cap a)$

$[1 ; B 16 ; B 10]$

$$
=((a \cap a) \cup b) \cap a=(a \cup b) \cap a
$$

$[B 12, c / a ; B 10]$

$B 19[a b]: a, b \in A . \supset . a=a \cap(a \cup b)$

$[B 18 ; B 17, a / a \cup b, b / a]$

B20 [ab]: $a, b \in A . \supset . a \cup b=b \cup a$

PR $[a b]: \mathrm{Hp}(1) . \supset$.

$a \cup b=((b \cup a) \cap a) \cup((b \cup a) \cap b)$

$=(((b \cap(b \cup a)) \cup a) \cap(b \cup a)$

$[1 ; B 13 ; B 18, a / b, b / a]$

$$
=(b \cup a) \cap(b \cup a)=b \cup a
$$

$[B 12, a / b \cup a, b / a, c / b]$

B21 $[a b]: a, b \in A . a \cup b=b . \supset . a \cap b=a$

B22 $[a b]: a, b \epsilon^{\prime} A . a \cap b=a . \supset . a \cup b=b$

$D 1 \quad[a b] \therefore a, b \in A . \supset: a \leqslant b . \equiv a \cap b=a$

B23 $[a b] \therefore a, b \in A . \supset: a \leqslant b . \equiv a \cup b=b$

$B 24[a b]: a, b \in A . a \leqslant b . b \leqslant a . \supset . a=b$

$[B 19, a / b, b / a ; B 10, a / b \cup a]$

[B19]

$[B 17 ; B 16, a / b, b / a]$

$B 25[a b c]: a, b, c \in A . a \leqslant b . a \leqslant c . \supset . a \leqslant b \cap c$

[D1;B21;B22]

$[D 1 ; B 10]$

PR $[a b c]: H p(3) . \supset$.

4. $a \cup b=b$.

$[1 ; 2 ; B 23]$

5. $a \cap c=a$.

6. $\quad a \cup(b \cap c)=(a \cap c) \cup(b \cap c)=(c \cap a) \cup(c \cap b)$

$[1 ; 3 ; D 1, b / c]$

$[1 ; 5 ; B 17, b / c ; B 17, a / b, b / c]$ $=(c \cap b) \cup(c \cap a)=((a \cap c) \cup b) \cap c$

$[B 20, a / c \cap a, b / c \cap b ; B 11, a / c, c / a]$ $=(a \cup b) \cap c=b \cap c$.

$a \leqslant b \cap c$

B26 $[a b c]: a, b, c \in A . a \leqslant c . b \leqslant c . \supset . a \cup b \leqslant c$

$[1 ; B 23, b / b \cap c ; 6]$

PR $[a b c]: \mathrm{Hp}(3) . \supset$.

4. $a \cap c=a$.

$[1 ; 2 ; D 1, b / c]$

5. $b \cap c=b$.

6. $(a \cup b) \cap c=(b \cup a) \cap c=((b \cap c) \cup a) \cap c$

$$
\begin{aligned}
& =(c \cap a) \cup(c \cup b) \\
& =(a \cap c) \cup(b \cup c)=a \cup b .
\end{aligned}
$$

$a \cup b \leqslant c$

$B 27[a b]: a, b \in A . \supset . a \cap b \leqslant a$

B28 $[a b]: a, b \in A . \supset . a \cap b \leqslant b$

$B 29[a b]: a, b \in A$.ग. $a \leqslant a \cup b$

$B 30[a b]: a, b \in A . \supset . a \leqslant b \cup a$

$[1 ; 3 ; D 1, a / b, b / c]$

$[1 ; B 20 ; 5]$

$[B 12, a / c, b / a, c / b]$

$[B 17, b / c ; B 17, a / c ; 4 ; 5]$

$[1 ; D 1, a / a \cup b, b / c ; 6]$

$[B 16 ; B 23, a / a \cap b, b / a]$

$[B 27, a / b, b / a ; B 17]$

$[B 19 ; D 1, b / a \cup b]$

[B29; B20]

$B 31[a b c]: a, b, c \in A . \supset . c \leqslant a \cup(b \cup c)$

PR $[a b c]: \operatorname{Hp}(1) . \supset$.

2. $c \cap(a \cup(b \cup c))=(a \cup(b \cup c)) \cap c=c$.

$[1 ; B 17, a / c, b / a \cup(b \cup c) ; B 2, a / c, c / a]$

$c \leqslant a \cup(b \cup c)$

$[1 ; D 1, a / c, b / a \cup(b \cup c) ; 2]$

$B 32[a b c]: a, b, c \in A . \supset . b \leqslant a \cup(b \cup c)$

$[B 31, b / c, c / b ; B 20, a / c, c / b]$ 
B33 $[a b c]: a, b, c \epsilon A . \supset . a \leqslant(a \cup b) \cup c$

$B 34[a b c]: a, b, c \in A . \supset . b \leqslant(a \cup b) \cup c$

$[B 32, a / c, b / a, c / b ; B 20, a / c, b / a \cup b]$

$B 35[a b c]: a, b, c \in A . \supset .(a \cup b) \cup c=a \cup(b \cup c)$

PR $[a b c]: \operatorname{Hp}(1) . \supset$.

2. $a \cup b \leqslant a \cup(b \cup c)$.

$[1 ; B 26, c / a \cup(b \cup c) ; B 29, b / b \cup c ; B 32]$

3. $(a \cup b) \cup c \leqslant a \cup(b \cup c) . \quad[1 ; B 26, a / a \cup b, b / c, c / a \cup(b \cup c) ; 2 ; B 31]$

4. $b \cup c \leqslant(a \cup b) \cup c$.

$[1 ; B 26, a / b, b / c, c /(a \cup b) \cup c ; B 34 ; B 30, a / c, b / a \cup b]$

5. $\quad a \cup(b \cup c) \leqslant(a \cup b) \cup c$.

$[1 ; B 26, b / b \cup c, c /(a \cup b) \cup c ; B 33 ; 4]$

$(a \cup b) \cup c=a \cup(b \cup c)$

$[1 ; B 24, a /(a \cup b) \cup c, b / a \cup(b \cup c) ; 3 ; 4]$

B36 $[a b c]: a, b, c \in A . a \leqslant b . b \leqslant c . \supset . a \leqslant c$

PR $[a b c]: \operatorname{Hp}(3) . \supset$.

4. $a \cup b=b$.

$[1 ; B 23 ; 2]$

5. $b \cup c=c$.

6. $a \cup c=a \cup(b \cup c)=(a \cup b) \cup c=b \cup c=c$. $a \leqslant c$

$B 37[a b c]: a, b, c \epsilon A . \supset .(a \cap b) \cap c=a \cap(b \cap c)$

$[B 33, a / b, b / a ; B 20]$

PR $[a b c]: \mathrm{Hp}(1) . \supset$.

2. $(a \cap b) \cap c \leqslant a$.

$[1 ; B 36, a /(a \cap b) \cap c, b / a \cap b, c / a ; B 27, a / a \cap b, b / c ; B 27]$

3. $(a \cap b) \cap c \leqslant b$.

$[1 ; B 36, a /(a \cap b) \cap c, b / a \cap b, c / b ; B 27, a / a \cap b, b / c ; B 28]$

4. $(a \cap b) \cap c \leqslant b \cap c$.

$[1 ; B 25, a /(a \cap b) \cap c ; 3 ; B 28, a / a \cap b, b / c]$

5. $(a \cap b) \cap c \leqslant a \cap(b \cap c) . \quad[1 ; B 25, a /(a \cap b) \cap c, b / a, c / b \cap c ; 2 ; 4]$

6. $\quad a \cap(b \cap c) \leqslant b$.

$[1 ; B 36, a / a \cap(b \cap c), b / b \cap c, c / b ; B 28, b / b \cap c ; B 27, a / b, b / c]$

7. $\quad a \cap(b \cap c) \leqslant c$.

$[1 ; B 36, a / a \cap(b \cap c), b / b \cap c ; B 28, b / b \cap c ; B 28, a / b, b / c]$

8. $\quad a \cap(b \cap c) \leqslant a \cap b . \quad[1 ; B 25, a / a \cap(b \cap c), b / a, c / b ; B 27, b / b \cap c ; 6]$

9. $a \cap(b \cap c) \leqslant(a \cap b) \cap c . \quad[1 ; B 25, a / a \cap(b \cap c), b / a \cap b ; 8 ; 7]$ $(a \cap b) \cap c=a \cap(b \cap c) \quad[1 ; B 24, a /(a \cap b) \cap c, b / a \cap(b \cap c) ; 5 ; 9]$

B38 $[a b c]: a, b, c \in A . a \leqslant c . \supset . a \cup(b \cap c)=(a \cup b) \cap c$

PR $[a b c]: H p(2) . \supset$.

3. $a \cap c=a$.

$a \cup(b \cap c)=(a \cap c) \cup(b \cap c)=(c \cap a) \cup(c \cap b)$

$[1 ; D 1, b / c ; 2]$

$[1 ; 3 ; B 17, b / c ; B 17 ; a / b, b / c]$

$$
=(c \cap b) \cup(c \cap a)
$$

$=((a \cap c) \cup b) \cap c=(a \cup b) \cap c$

$[B 20, a / c \cap a, b / c \cap b]$

$[B 12, a / c, c / a ; 3]$

Since formulas $B 11, B 10, B 20, B 17, B 35, B 37, B 15, B 19$, and $B 38$ are the consequences of $B 1$ and $B 2$, it is proved that axioms $B 1$ and $B 2$ can be accepted as a postulate-system for modular lattices.

2 The mutual independence of axioms $B 1$ and $B 2$ is established by using the following algebraic tables: 
酷

\begin{tabular}{l|lll|ll}
$\cap$ & $\alpha$ & $\beta$ & $\cup$ & $\alpha$ & $\beta$ \\
\hline$\alpha$ & $\alpha$ & $\beta$ & $\alpha$ & $\alpha$ & $\alpha$ \\
$\beta$ & $\alpha$ & $\beta$ & $\beta$ & $\alpha$ & $\alpha$
\end{tabular}$;$

An:

\begin{tabular}{l|lll|ll}
$\cap$ & $\alpha$ & $\beta$ & $\cup$ & $\alpha$ & $\beta$ \\
\hline$\alpha$ & $\alpha$ & $\alpha$ & $\alpha$ & $\alpha$ & $\alpha$ \\
$\beta$ & $\alpha$ & $\beta$ & $\beta$ & $\alpha$ & $\alpha$
\end{tabular}

which are given by Kolibiar in [1], pp. 385-386.

Namely:

(a) verifies $B 2$, but falsifies $B 1$ for $a / \beta, b / \beta$, and $c / \beta$ : (i) $(\beta \cap \beta) \cup$ $(\beta \cap \beta)=\beta \cup \beta=\alpha$, (ii) $((\beta \cap \beta) \cup(\beta \cup \beta)) \cap \beta=(\beta \cup \alpha) \cap \beta=\alpha \cap \beta=\beta$.

(b) verifies $B 1$, but falsifies $B 2$ for $a / \beta, b / \beta$, and $c / \beta$ : (i) $\beta=\beta$, (ii) $(\beta \cup(\beta \cup \beta)) \cap \beta=(\beta \cup \alpha) \cap \beta=\alpha \cap \beta=\alpha$.

Thus, the proof of $(A)$ is complete.

NB After this paper was composed I learned that Kolibiar's problem had already been solved by J. Riečan, $c f_{.}$, [2], who proved that formulas $B 12$ and $B 2$, given above, constitute an adequate postulate-system for modular lattices. It should be noted that Riečan's system is shorter than mine.

\section{REFERENCES}

[1] Kolibiar, M., "On the axiomatic of modular lattices," in Russian, Czechoslovak Mathematical Journal, v. 6 (81) (1956), pp. 381-386.

[2] Riečan, J., “'Zu der Axiomatik der modulären Verbände," Acta Facultatiae Nationalis Universitatis Comenianensis, Mathematica, v. 2 (1958), pp. 257-262.

University of Notre Dame

Notre Dame, Indiana 